cus above. Abdomen elsewhere resonant. Pulse 126, bounding, full. I ordered hot flannels to the abdomen; perfect rest in the recumbent posture; the bed-pan to be always used rather than the night-stool; a pillow to be placed beneath the girl's knees, so as to permit relaxation of the muscles of the abdominal wall; and only milk (as much as two pints in the day) for food. Also to take half an ounce of the following mixture every four hours: two scruples of compound chalk powder with opium, two drachms of compound tincture of cardamoms, a drachm of spirit of chloroform, and four ounces of water.-8.30 P.M. : She has vomited one of two doses as yet taken. Bowels not again opened. Urine dark-coloured, and containing a sediment of urates. Pulse 126 ; respiration 33 ; temperature $101 \cdot 4{ }^{\circ}$. Belly more tender. I discovered now a distinct tumour in the right iliac region, which occupied much the position of the cæcum, except that it extended a little to the left of the middle line just above the pubes, and reached upwards quite to the level of the umbilicus over the region of the ascending colon.

26th. - 8.30 A.M.: The patient has passed a oleepless night, vomiting everything. Pulse 126, full, not wiry (as in peritonitis); respiration 26 ; temperature $101 \cdot 3^{\circ}$. Bowels not opened; a very little urine passed, containing thick lateritious sediment. Pain paroxysmal; abdomen more swollen and tympanitic, except over the region of the tumour, and too tender to allow of prolonged manipulation. This general tympanitic tumefaction, apart from the tumour, is clearly prodnced by swelling of the small intestines; for it is most prominent around and below the umbilicus, whilst over the colon there is no enlargement. I now surmised (for reasons hereafter stated) that probainly the case was one of ileo-cæcal intussusception.-9.50 A.乃工: Dr. Habershon, being summoned in consultation, confirmed the diagnosis; and, when the overlying abdominal muscles were relaxed by flexion of the right thigh, could distinguish that the tumour was of somewhat cylindrical shape, immovable, and of firm consistence. He ordered the following medicine: Spirit of chloroform, a drachm and a half; solution of hydrochlorate of morphia, one drachm; syrup of lemons, three drachms; to four ounces of water: half an ounce to be taken every three hours. I proposed to him to inflate the colon with air. Dr. Habershon wished the morphia to be first fully tried, but thought that if no benefit had occurred in twelve hours' time inflation might be employed.-6.30 P.M.: Pulse 108; pupils somewhat contracted. There is scarcely any abatement of the urgent symptoms, although the mixture has been retained upon the stomach.9.30 P.M. : Accompanied by my friend Dr. Easton, I proceeded to inflate per rectum. The patient, disliking chloroform, consented to brave the operation without it. I then slowly pumped-in air until the abdomen over the colon was tensely swollen, when the patient almost swooned from pain, and $D x^{2}$. Easton and myself deemed it prudent to desist. The backward pressure from the bowel of the air in the instrument was, in fact, so great as to lift the piston upon the removal of my hand. Medicine to be continued, and cold milk taken during night.

27th.-8.30 A.M. : Pulse 116 ; respiration 23 ; temperature $101 \cdot 3^{\circ}$. All the symptoms of obstruction remain; the vomited matters have a foul smell. Pupils a little contracted; patient feeble. She dozed during the night, but was awoke by paroxysms of pain. Tongue loaded with white creamy fur; thirst great, but everything swallowed is at once rejected. - 2.30 P.Mr. : No relief having been obtained, I gradually injected, per rectum, about forty-five ounces of lukewarm water, by means of an ordinary enema syringe, so that full distension is produced. The water, gurgling in the bowel, is traceable in the descending colon to the tumour, which I manipulated as much as the patient could endure, hoping that thereby the water might the more readily restore the patency of the canal. I kept the intestine thus distended for about fifteen minutes, but did not experience the sudden want of resistance to the hand holding the piston which is said to denote return of the intussusception. Just before withdrawal of the tube $I$ allowed about a pint and a half of water, that escaped almost colourless, to run away. -9.40 P.M. : Some change for the better has ensued. Pulse 102 ; respiration 22 ; temperature $100 \cdot 8^{\circ}$. She has vomited twice, the vomit consisting only of milk or other material just placed in the stomach. Pain decidedly less. To continue the same medicinal and dietetic treatment. 28th.-9.50 A.M. : She vomited once only during the night, when some beef-tea was given. Pulse 88 ; respiration 22; temperature $97 \cdot 6^{\circ}$. Pain no longer paroxysmal ; abdomen less distended, more supple; the region over which exists dulness upon percussion not diminished ; tongue cleaning at edges. She is much inclined to sleep. To take a dose of the morphia mixture every four hours only.

From this point progress to recovery was uninterrupted. She never vomited again.

29th.-Air begins to pass per rectum. Pulse 88; temperature $98 \cdot 2^{\circ}$.

May 2nd.-Bowels not yet opened, and none of the twelve to eighteen ounces of water which remained in the colon after the withdrawal of the enema-pipe on April 27th has escaped. Being anxious to discover if fæces had passed down to the rectum, I introduced my finger, and found hard scybalous masses distending the bowel.

3rd.-The patient has strained much three or four times: to empty the rectum, but the Iumps seem too hard and bulky to pass the sphincter. I therefore administered an enema of about ten ounces of lukewarm water. An hour afterwards the bowels were copiously relieved. The motion, contrary to my express injunctions, was thrown away by mistake, but the mother reported that no blood was detectible therein, nor any indigestible material that might have caused the obstruction.

At this time the swelling above the right groin was daily decreasing, though all traces thereof were not lost until May 6th. Dr. Habershon, to whom I communicated thiss fact, deemed the continuance of the swelling for these eight or nine days (after release of the intussusception) to be due to inflammatory thickening of the mesentery, peritoneum, and coats of the bowel, which necessarily could not return to the natural condition immediately that the invagination ceased. The intestine, at the seat of lesion, was probably also paralysed for a few days-a condition favourable to detention of ingesta at the spot.

During the whole period of recovery no purgative was allowed; fluid diet (milk especially, in regulated quantities) was given for several days ; the morphia mixture, though in daily diminished doses, was continued until May 4th; and all other means were for some time adopted, in order to give the intestine " therapeutical rest."

( $T o$ be concluded.)

\section{CASE OF CYST WITHIN THE EYE.}

\section{By C. K. FISKE, EsQ.}

THE following account of a cyst within the eye, of very unusual occurrence, will be of interest to the readers of The Lancet.

Mr. E. P_, of Hillsboro', County of Albort, N.B., aged forty-six, called on me in the winter of $1862-3$ for advice in regard to his left eye, which had been irritable and painful for several weeks, and was fast becoming blind. On hastily examining the eye, I at once discovered what I thought to be the lens dislocated, and lying in the anterior chamber directly in front of the pupil, nearly covering that aperture, leaving a crescent-shaped portion of it open in the upper part, through which there was imperfect vision. I immediately told my patient that the lens was dislocated, when he informed me that he had that day consulted Dr. Tyrrell (son of the late ophthalmic suroeon of that name), who gave the same opinion. At a later hour in the day the patient called for a more careful examination of the case, when I found the crystalline lens quite healthy, and in its natural position. The expansion of the pupil under the influence of atropia enabled the patient to see distinetly enough to read fine print at the same focal distance as with the right eye, and I found the object in the anterior chamber to be a cyst attached to the lower ciliary margin of the iris. It being a rather anomalous case, I proposed a consultation with Dr. Tyrrell, which was readily granted, and he agreed with me in my diagnosis. We then decided to remove as much of the cyst from the eye as practicable through a small opening in the cornea, and accordingly Dr. Tyrrelf made a slight cut with a cataract-knife, and with a small 
hook drew out some shreds of the cyst, and cut them off with soissors. The wound healed in a day or two, and the eye assumed a natural and healthy appearance, with good vision.

On further inquiry into the history of the case, $I$ learned that forty years previously, when a boy six years of age, the patient had received a wound precisely in the locality of the attachment of the cyst with a sharp-pointed dart whilst at play, which caused much irritation for several days, after which there was no more trouble with the eye till forty years later, and a few weeks before seeking my advice.

On the 20th of the following May the patient was in town again with the cyst refilled, the eye irritable, and sight obscured. The semi-transparent body in the anterior chamber had very much the appearance of a hydatio, with its proboscis turned downwards, with the exception of being attached to the old cicatrix of the wound, while the proboscis of a living cysticercus is so placed by gravitation. At this time I contented myself with only puncturing the cyst with a cataract needle, and allowing it to collapse, when the eye assumed its natural appearance, and the pain and intolerance of light passed off. The eye remained comfortable till the following September, when the cyst again filled up, giving pain and indistinct vision as before.

On the 3rd of September I removed the whole of the cyst through a small puncture in the cornea by means of a minute canula forceps. This gave great relief, and promised to be more effectual than anything done before ; and, in fact, there was but little trouble with the eye till three years later, Sept. 1866, when it again became very painful. Puncture of the cornea and cyst gave relief, but for a few days at a time.

There came on great pain in the eye and brow, and in the latter part of October the iris and lower portion of the cornea became involved with what had been the base of the cyst, and vision was quite extinct; the patient also had lost much flesh, and had not been able to attend to business. I then advised excision of that portion of the eye diseased; in fact, the whole of the cornea, iris, and lens.

In a few weeks the patient returned in great distress, determined to submit to excision of the whole eye, if necessary, rather than suffer longer.

On the 24th November I placed him under the influence of chloroform, and removed nearly the anterior third of the globe, with great relief to the patient; and in a few weeks he was able to attend to business with comfort, having gained thirty pounds of flesh in the mean time. In the following spring he was fitted with an artificial eye, and his health remains good at the present date.

This case seems analogous to one reported by Mr. James Dixon, F.R.C.S., and first seen and treated by Mr.Dalrymple, which was frequently under surgical treatment from 1846 till June 1856, and may have required further treatment subsequently. Both were caused by punctured wounds near the junction of the cornea and sclerotica, penetrating to the ciliary margin of the iris; and both became troublesome after many years of rest. The morbid action in one case was set up forty years after the receipt of the wound, and in the other case twelve years. In both the cornea remained remarkably transparent through much pain and irritation and many operations for evacuating the cyst. Mr. Dixon speaks of his case as unlike anything he had met with in his practice, or seen recorded as occurring in that of others.

St. John, New Brunswick, Aug. 1869.

\section{ON A CASE OH}

\section{INDUCTION OF PREMATURE LABOUR.}

\section{By LEWIS C. NANNEY,}

ASSISTANT-SURGEON, H.M.'S MADRAS ARMX.

Ir is not necessary for me, as a matter of course, to enter into details regarding the importance of obstetric operations in general, as we already know the dangers to which both mother and child are subjected during these operations, and at the same time the immense risk we incur by not acting promptly when interference is necessary-i.e., that nature, if left to herself, will not perform what we require of her.

The following case will, I trust, prove interesting, as the treatment adopted was attended with the most marked success.

Mrs. — aged thirty, a small woman, of an anæmic appearance, consulted me immediately on my arrival at this station. The history of the case is as follows:-Mrs. has had two children, and on both occasions craniotomy was performed. She has resided for about eighteen years in India, and, of course, her general health has suffered considerably from the enervating nature of the climate. The early stage of her two former labours appeared to go on satisfactorily enough for a short time, but uterine contractions became feeble after a few hours, and at last entirely ceased, whilst the head was completely above the brim of the pelvis. Symptoms of prostration rapidly supervened, and, after waiting for twenty-four hours, it was considered by the medical officers attending her that further delay might prove serious. Craniotoniy was performed. She had an attack of phlegmasia dolens after each labour.

She consulted me when she had completed her sixth month. My first object was to improve her general health, which had become much impaired during a long and tedious march in the rainy season. I recommended regular exercise, stimulants, good generous diet, combined with iron and quinine. Taking into consideration the history of her two former labours, and the risk $I$ should incur by allowing the patient to go on to full time, I decided at once upon inducing premature labour at the end of the seventh month, and considered myself perfectly justified in doing so ; for the patient herself was particularly anxious to have a living child, and the risk of inducing premature labour at the end of the seventh month would not be attended with so much danger to the patient as the performance (in all probability) of an operation for the third time, in addition to saving the life of the child-at any rate, of giving it a much better chance.

On the morning of the 2nd of October I commenced the operation for the induction of premature labour (the patient having completed her seventh month) by introducing a small sponge tent, and retaining it in its position by a plug in the vagina. This was removed every six hours, and plugs of larger sizes introduced, tepid water being thrown up the vagina occasionally, for the purpose of enlarging the sponge. This plan of treatment was pursued for three days, and dilatation gradually began to take place.

On the morning of the 5th inst., the strength of the patient having somewhat diminished, I decided on puncturing the membranes, which I had delayed resorting to in order to afford as much protection as possible to the child. They were easily reached with the finger, and an unusually large quantity of liquor amnii made its escape. The os uteri was then dilated to about the size of a florin. I administered thirty-minim doses of liquid extract of ergot of rye every quarter of an hour for the space of one hour. Pains commenced, and continued at intervals until 7 P.M., when they gradually began to diminish. Every effort on my part was made to keep up the strength of the patient; strong chicken broth and Madeira were given three or four times during the day. At 8 P.M., her pulse, which was naturally quick, rapidly increased in frequency, but diminished in fulness and strength. The head of the child was then entering the cavity of the pelvis, and the pains beino inadequate, I decided upon terminating the labour with as little delay as possible. A small quantity of chloroform was administered, the forceps applied, and in a few minutes $I$ succeeded in bringing into the world a living child. The antero-posterior diameter of the pelvis was contracted in size, so that a child of seven months passed with considerable difficulty. What would then have been the result had I allowed the patient, as on previous occasions, to go on to the end of her full time?

I omitted to mention that, during her first labour, rupture of the perineum took place, followed by sloughing. There was no after-hæmorrhage. The placenta came away without any difficulty.

This is the fifth day since delivery, and mother and child are both doing well.

Hingolee, East Indies, Oct. 9th, 1869.

Froy the "Norwich Argus" we learn that Dr. John T. Skximshire, of Holt, has received the handsome present of a silver cream-jug from " 62 poor and grateful patients of Holt and Letheringsett." 